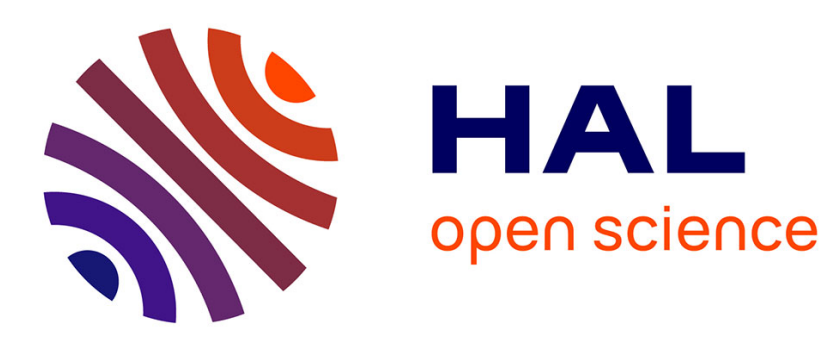

\title{
LAR Image transmission over fading channels: a hierarchical protection solution
}

Wassim Hamidouche, Christian Olivier, Marie Babel, Olivier Déforges, Hervé Boeglen, Pascal Lorenz

\section{- To cite this version:}

Wassim Hamidouche, Christian Olivier, Marie Babel, Olivier Déforges, Hervé Boeglen, et al.. LAR Image transmission over fading channels: a hierarchical protection solution. The Second International Conference on Communication Theory, Reliability, and Quality of Service, Jul 2009, Colmar, France. pp.1-4. hal-00373284

\section{HAL Id: hal-00373284 https://hal.science/hal-00373284}

Submitted on 3 Apr 2009

HAL is a multi-disciplinary open access archive for the deposit and dissemination of scientific research documents, whether they are published or not. The documents may come from teaching and research institutions in France or abroad, or from public or private research centers.
L'archive ouverte pluridisciplinaire HAL, est destinée au dépôt et à la diffusion de documents scientifiques de niveau recherche, publiés ou non, émanant des établissements d'enseignement et de recherche français ou étrangers, des laboratoires publics ou privés. 


\section{LAR IMAGE TRANSMISSION OVER FADING CHANNELS: A HIERARCHICAL PROTECTION SOLUTION}

\author{
W. HAMIDOUCHE, C. OLIVIER \\ Laboratoire SIC XLIM, UMR CNRS \\ 6172 \\ Téléport 2, Bd Marie et Pierre Curie \\ 86962 Futuroscope Cedex France \\ hamidouche@sic.sp2mi.univ- \\ poitiers.fr
}

\author{
M. BABEL, O.DEFORGES \\ IETR UMR CNRS 6164 - INSA de \\ Rennes, \\ 20 av. des Buttes de Coësmes - CS \\ 14315 \\ 35043 Rennes, France
}

\author{
Hervé BOEGLEN, Pascal LORENZ \\ Laboratoire MIPS, Equipe GRTC \\ IUT de Colmar \\ 34 rue du Grillenbreit, \\ 68008 Colmar Cedex France \\ herve.boeglen@uha.fr
}

\begin{abstract}
The aim of this paper is to present an efficient scheme to transmit a compressed digital image over a non frequency selective Rayleigh fading channel. The proposed scheme is based on the Locally Adaptive Resolution (LAR) algorithm, and the Reed-Solomon error correcting code is used to protect the data against the channel errors. In order to optimize the protection rate and ensure better protection we introduce an Unequal Error Protection (UEP) strategy, where we take the hierarchy of the information into account. The digital communication system also includes appropriate interleaving and differential modulation.

Simulation results clearly show that our scheme presents an efficient solution for image transmission over wireless channels, and provides a high quality of service, outperforming the JPWL scheme in high bit error rate conditions.
\end{abstract}

Keywords: Fading channels, Locally Adaptive Resolution (LAR), scalability, Unequal Error Protection (UEP), (JPWL).

\section{INTRODUCTION}

Nowadays, wireless communications and their applications are undergoing major expansion and have captured a great attention of media attention as well as the imagination of the public. However, wireless channels are known to generate a high number of errors which perturb complex multimedia applications such as image or video transmission. For these reasons, designing a suitable system for image transmission over wireless channel remains a major issue.

A number of solutions have already been designed for fixed image and video transmission over wireless channels. In [1], the SPIHT image coding scheme is protected by using product codes which consist of concatenated RCPC/CRC and Reed-Solomon codes. The protection of SPIHT bitstream can also be achieved using both an UEP strategy and an iterative process to optimize these product codes [2]. A scheme dedicated to JPEG2000 transmission over a wireless channel has been described in [3]: optimized product codes consisting of turbo codes and ReedSolomon codes have been implemented for that purpose. JPEG2000 wireless (JPWL) [4] was tested over a WCDMA wireless channel [5], through a memoryless Binary Symmetric Channel (BSC) and Gilbert-Elliot channel in [6]. All these solutions need low code rates ranging from $1 / 3$ to $2 / 3$ and a scalable compression algorithm. The scheme proposed in this paper relies on a scalable version of the Locally Adaptive Resolution (LAR) image coder [7], named Interleaved S+P. The main characteristics of the Interleaved $\mathrm{S}+\mathrm{P}$ algorithm, based on the LAR coding method, are lossy and lossless compression modes, and high scalability together with advanced services such as progressivity in representation and transmission and Region Of Interested (ROI) coding. Moreover, Interleaved S+P algorithm offers better coding results than JPEG2000 lossless mode. In order to provide robust LAR code streams and high code rate, we exploit the intrinsic scalability of Interleaved S+P coder together with a UEP strategy.

This paper is structured as follows: both Rayleigh channel constraints and the methods based on differential modulation used to circumvent them are presented in section II. Section III details the scalable Interleaved S+P solution. A UEP strategy applied to the LAR codestream with Reed-Solomon codes is described in section IV. Experimental results with LAR and JPWL are reported in section $\mathrm{V}$, and finally conclusions are given in section VI.

\section{FADING CHANNELS}

In mobile radio communications, the electromagnetic waves often do not reach the receiving antenna directly because of obstacles blocking the line-of-sight path. In fact, the received waves consist of a superposition of waves coming from all directions: this property is essentially due to reflection, diffraction and scattering caused by buildings, trees and other obstacles. This effect is known as multipath propagation [8]. As a result, the received signal is equal to the sum of attenuated, delayed, and phase-shifted replicas of the transmitted signal, with each replica affecting the others. Depending on the phase of each partial wave, the superposition can be constructive or destructive. Moreover, as far as of digital transmission is concerned, the shape of the transmitted impulse itself can be distorted. In this case, because of multipath propagation, several individually distinguishable impulses can be detected at the receiver. This effect is called impulse dispersion. Impulse distortion depends on propagation delay differences and on the relationships between of the partial waves. The consequence of this effect is a distortion of the frequency response characteristic of the transmitted signal.

Besides multipath propagation, the Doppler effect has also a negative influence on the transmission characteristics of the mobile radio channel [8]. Because of the receiver's mobility, the Doppler effect causes a frequency shift of each partial wave. As a result, the spectrum of the transmitted signal undergoes frequency expansion during transmission. This effect is called frequency dispersion. The value of the frequency dispersion mainly depends on the maximum Doppler frequency and the amplitudes of the received partial waves. In the time domain, the 
Doppler effect implies that the impulse response of the channel becomes time-variant. Multipath propagation, combined with the movement of the receiver and/or the transmitter leads to drastic and random fluctuations in the received signal. Fades of 30 up to $40 \mathrm{~dB}$ and more below the mean value of the received signal level can occur several times per second.

In digital data transmission, the momentary fading of the received signal causes burst errors i.e. errors with strong connections with each other. Therefore, a fading interval produces burst errors in which burst length is determined by the duration of the fading. Given this situation, the development of efficient error protection for digital transmission systems naturally implies in-depth knowledge of the fading channel statistics.

\section{A. Statistical fading channel models}

Choosing a transmission technique and designing a digital receiver require the simulation of the effects of a fading channel. Since the pioneering work of Clarke [9] in the late sixties, a lot of theoretical works has been completed through measurement testing showing that, in the case of non frequency-selective channels, the fluctuations in the received signals can be modelled by multiplying the transmitted signal by an appropriate stochastic model process. In this paper we use the set of COST207 channel models established for the deployment of second-generation mobile telephony [10] and from this set we choose the COST207 TU (Typical Urban) channel model.

\section{B. Differential modulation Star 16QAM}

Differential modulation/demodulation techniques have proved to be very robust because they can mitigate the phase distortions caused by fading. Moreover, as no channel estimation is needed, system complexity is reduced significantly. In this application, we use a high spectral efficiency 16-point constellation that may be differentially encoded and decoded. This scheme is known as Star 16QAM (Quadrature Amplitude Modulation) [11]. Star 16QAM uses a combination of independent 2DASK (Differential Amplitude Shift Keying) and 8DPSK (Differential Phase Shift Keying). Three of the four bits are modulated by a Gray encoded 8DPSK. The remaining bit is amplitudemodulated by a 2DASK scheme.

\section{INTERLEAVED S+P: SCALABLE IMAGE CODING}

This section describes the LAR (Locally Adaptive Resolution) method. The scalable version of this image codec is specified and the associated functionalities are presented.

\section{A. Flat LAR Coder}

The LAR compression method is a two-layer codec: a flat codec and a complementary spectral one. Images can be considered as a superposition of global information and texture (local information). The flat LAR codec is designed to provide a low bit-rate compressed image, whereas the spectral one encodes textures [7].

The basic idea is that local resolution, in other words pixel size, can depend on local activity. This leads to the construction of a variable-resolution image based on a quadtree data structure. Thanks to this type of block decomposition, the size implicitly gives the nature of the block. Small blocks are naturally located on contours whereas large ones are situated in smooth areas. In a lossy context, this image content information controls a quantization of the luminance that corresponds to visual perception: large blocks require fine quantization (in uniform areas, human vision is strongly sensitive to brightness variations) while coarse quantization (low sensitivity) is sufficient for small blocks.

\section{B. Scalable LAR coder: the Interleaved S+P}

The "Interleaved S+P" algorithm is designed to provide a fully scalable representation of images [12]. This new method keeps the same main structure as the Flat LAR method ( $\$ 3.1$ ), namely a two-layer codec, so that the first part of the compressed bitstream allows for the reconstruction of the LAR block image. The Interleaved S+P outperforms state-of-the-art methods both in terms of lossless and lossy compression. The performance of our algorithm stems from the initial quadtree partition. As it is perfectly correlated to local activity, the method uses the information to adapt the prediction process in such a way as to decrease coding cost. Moreover, we split the bitstreams into different substreams according to the nature, location and local activity of the corresponding pixel. Implicit context modeling is then used, reducing the final entropy.

\section{Functionalities}

\section{1) Scalable representation}

The pyramidal description of the images resulting from Interleaved $\mathrm{S}+\mathrm{P}$ encoding provides various scalability levels. The conditional decomposition (the constraint of two successive descent processes by the initial quadtree partition of the image) provides a highly scalable representation in terms of both resolution and quality. Figure 1 shows an example of these LAR method characteristics. This feature is definitely a major advantage for applications such as remote database consultation or other advanced client-server solutions.

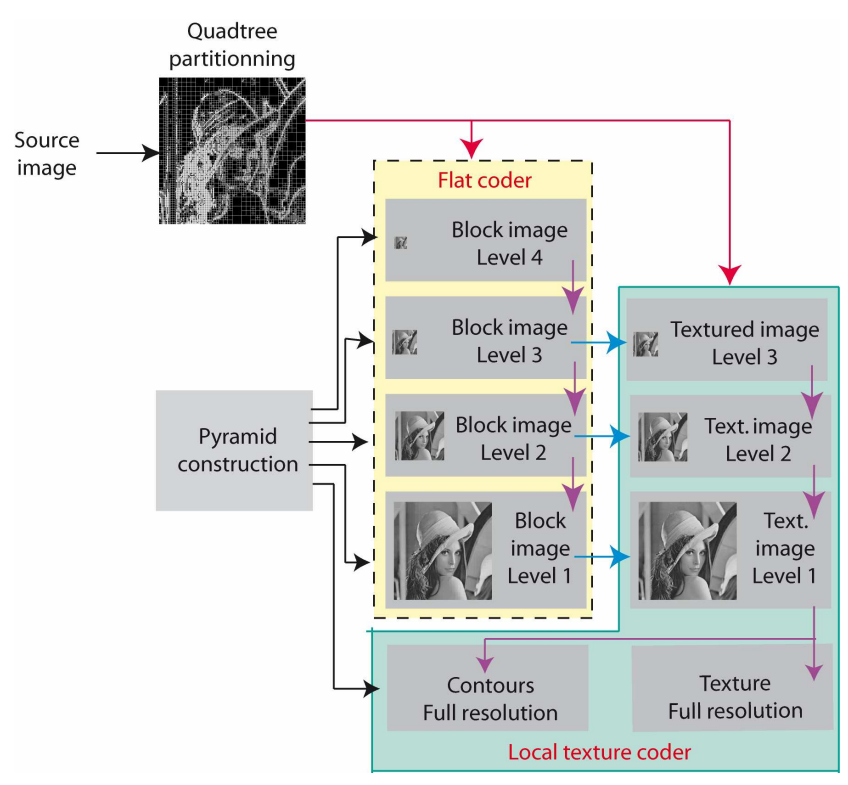

Figure 1. Interleaved $\mathrm{S}+\mathrm{P}$ and Scalable coding 


\section{2) Hierarchy and Robustness}

When errors occur during transmission, visual impact is dependant on the error location in the bitstream. The Interleaved $\mathrm{S}+\mathrm{P}$ method introduces intrinsic hierarchy that can be further exploited for cryptography or hidden data insertion purposes. In particular, the quadtree partition information and the highest level of the pyramid are mandatory for the reconstruction of any visually coherent image [13].

\section{HIERARCHICAL PROTECTION}

Limited bandwidth and distortions are the main features of a wireless channel. Therefore, both compression and secure transmission of sensitive data are simultaneously required. The LAR method and a UEP strategy are applied respectively here to compress and protect the original image. The UEP strategy takes account of the sensitivity of the ten substreams requiring protection and then optimizes the redundancy rate. The first class which contains both grid data and information resulting from level 4 of our representation is the most sensitive. The second one contains the substreams resulting from levels 3, 2 and 1 of the first pyramid and level 3 of the second one. Finally, the substreams corresponding to the levels 2 and 1 of the second pyramid, and the remaining substreams, namely the last level of each pyramid are assigned to the third class. The strategy of protection is then specifically defined for each class described previously: the code rate varies according to the level of sensibility of each class. More precisely, we allocate more channel symbols to the classes that carry important information and fewer to other classes.

In our application, we used the Reed Solomon Error Correcting Code (RS-ECC). We denote it as $\operatorname{RS}(n, k)$, where $n$ is the codeword symbol length and $k$ the number of the information symbol. The code associated with the first class is a $\operatorname{RS}(255,185)$ one which provides a high correction capability. The second class which contains the most important information about the image, is protected with an $\operatorname{RS}(255,192)$ code. The information carried by the third class is protected with an RS $(255,205)$ code. All these RS-ECCs are interleaved by using an appropriate block interleaver. This technique spreads the burst errors generated by the fading channel over a large number of symbols, so as not to exceed the RS code burst error capability. The diagram in Figure 2 summarizes the different layers of our transmission system.

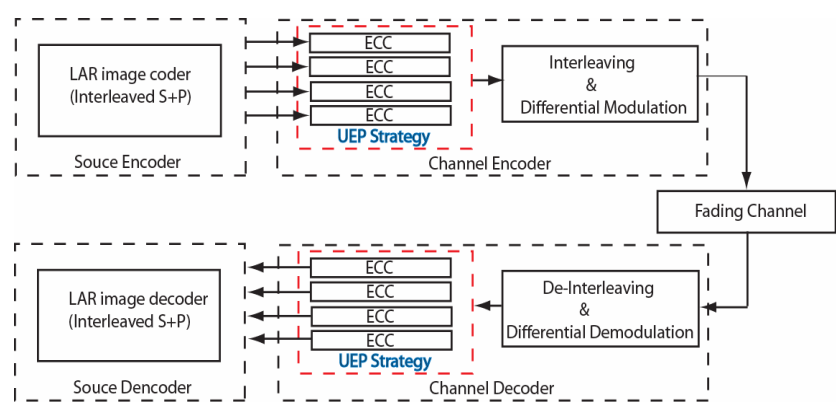

Figure 2: Overall layout of the multi-layers transmission/compression system.

\section{RESULTS}

In this section, we compare the performance of the LAR scheme with the standardized JPWL image coding system [6] over the non frequency selective fading channel. The transmission conditions we have adopted are those found on very limited bandwidth resources channels such as the one described in [14]. In those situations, the amount of ECC added to the image is an important factor: this is the reason why we adopt quite high rate ECC (typically R > 0.6).

The proposed layout for the transmission of Interleaved S+P streams over the COST207 TU channel was experimentally evaluated for the Lena image $(512 * 512)$. Without any error correction code, the compression rate was fixed at $0.32 \mathrm{bpp}$. Following the UEP strategy defined in section 4 , the overall protection rate chosen was $\mathrm{R}=0.77$.

For the JPWL coder, the Lena image was compressed into three layers: $0.1 \mathrm{bpp}$ for the base layer, $0.2 \mathrm{bpp}$ for the second layer, and $0.32 \mathrm{bpp}$ for the third layer. The overall protection rate is $\mathrm{R}=2 / 3$, and the selected Reed-Solomon codes are: $\mathrm{RS}(54,32)$ for the base layer, $\operatorname{RS}(46,32)$ for the second layer, and $\operatorname{RS}(43,32)$ for the third layer. One can notice that the protection is higher for the JPWL image $(\mathrm{R}=0.67)$ than it is for the LAR coded one $(\mathrm{R}=0.77)$. Using the same ECC, the ratio $\mathrm{R}$ can slightly vary from one image to another depending on its nature (the size of each layer).

The COST207 TU channel is characterized by two parameters: the Signal-to-Noise Ratio (SNR), and the Doppler frequency $f_{D}$ normalized to the symbol time $T_{S}\left(f_{D} \times T_{s}\right)$. The proposed system was optimized for a normalized Doppler frequency $f_{D} \times T_{s}=10^{-3}$ and for a variable SNR. The JPWL and LAR images were transmitted in exactly the same conditions.

Figure 3 shows variations of the received Peak Signal to Noise ratio (PSNR) versus Signal-to-Noise Ratio (SNR), when transmitting LAR and JPWL images over the COST207 TU channel. For the same SNR value but different channel characteristics, the visual quality of a reconstructed image can differ widely from one simulation to the other. This is the reason why each point in Figure 3 corresponds to an average of fifty simulations.

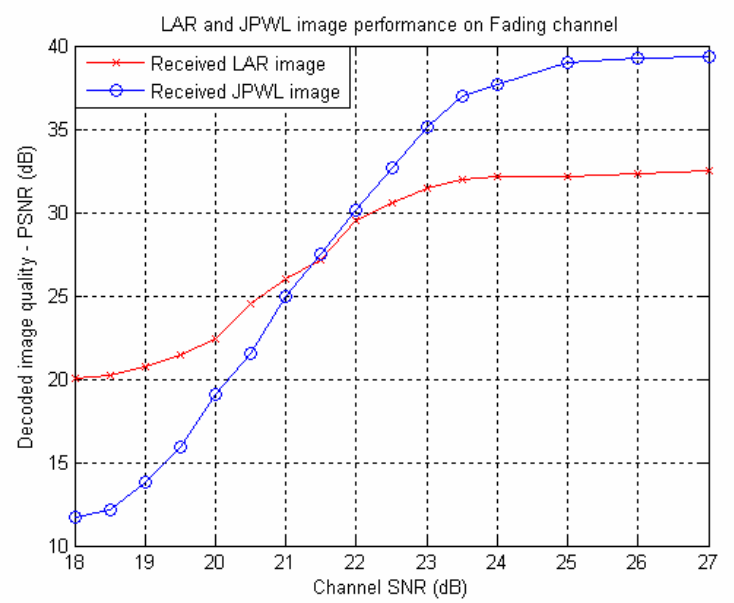

Figure 3: LAR and JPWL image performance through the COST207 TU channel.

Although the ECC protection adopted for the JPWL images is higher than that of the LAR images, Figure 3 clearly shows the superiority of the proposed layout in the case of bad transmission conditions $(\mathrm{SNR}<21 \mathrm{~dB})$. This observation is confirmed by the visual quality of images shown on Figure 4 . In 
the LAR image, the residual errors in the stream appear as an impulse noise on the reconstructed image. It can be easily discarded by applying simple post-processing such as a median filter. Such post-processing improves our results by approximately $3 \mathrm{~dB}$ and produces a better visual quality.

With a very high amount of binary errors, JPWL is unable to provide any visually acceptable reconstructed image (Figure 4). For intermediate SNR values $(21.5 \mathrm{~dB})$, if the PSNR of the received images are comparable, the visual quality of the LAR image still remains higher than JPWL (Figure 5). However, when the value of SNR exceeds $22 \mathrm{~dB}$, the JPWL scheme yields better results in terms of PSNR, whereas it is difficult to notice any difference between LAR and JPWL received images (Figure 6).

These results can be easily explained. Firstly, although objective metric PSNR is widely used in the image coding community, its results are removed from subjective quality assessments. In particular, the lowest block resolution of the LAR image is here about $2 \times 2$, eventually implying a natural contour shift that is imperceptible to the human eye but dramatic for a PSNR measure. Secondly, for the LAR scheme, no synchronization mechanisms were added, contrary to the JPWL layout. Because of the entropy coding stage (arithmetic coder), one error bit that is not corrected leads to the total desynchronization of the rest of the substream. A simple synchronization solution would increase the quality level without over-increasing the bit rate.

Finally, if we compare the amount of transmitted data, JPWL requires a higher quantity of bits for the same payload. If we use the same bit rate for the JPWL as for the LAR, we obtain even worse results for JPWL in terms of visual quality.

\section{CONCLUSION}

A novel image transmission scheme was proposed for compressed LAR image streams through frequency non selective fading channels. The intrinsic scalability provided by the Interleaved $\mathrm{S}+\mathrm{P}$ coder makes it possible to use an UEP strategy which takes the sensitivity of each substream into account. They are then more resilient to error by matching the code rate to sensitivity and a high code rate. The transmission results showed that our system provides a good quality of service when BER is lower then $2 \times 10^{-2}(\mathrm{SNR}<18 \mathrm{~dB})$ and clearly outperforms JPWL in poor transmission conditions even at lowest ECC protection level.

Acknowledgment: This work is supported by the French National Research Agency as part of the CAIMAN project.

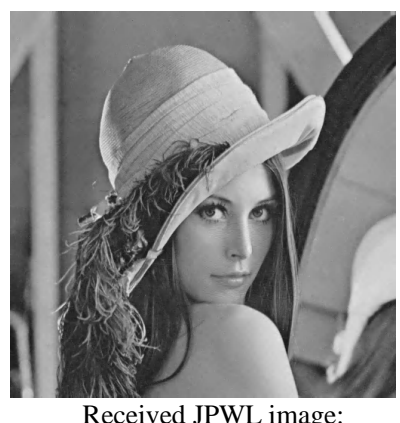

$38.95 \mathrm{~dB}$

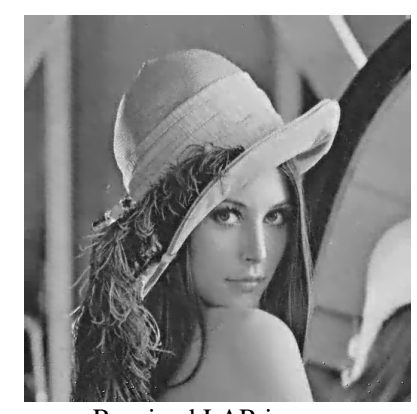

Received LAR image: $32.19 \mathrm{~dB}$
Figure 6: LAR and JPWL image transmission through the COST207 TU channel, $\mathrm{SNR}=25 \mathrm{~dB}, \mathrm{f}_{\mathrm{D}} \times \mathrm{T}_{\mathrm{s}}=0.001$, $\mathrm{BER}=3.5 \times 10^{-3}$
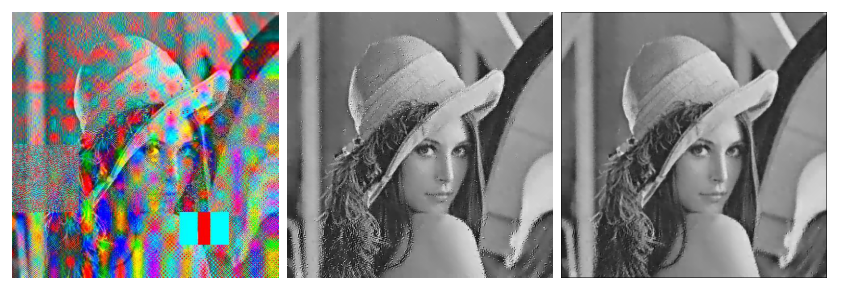

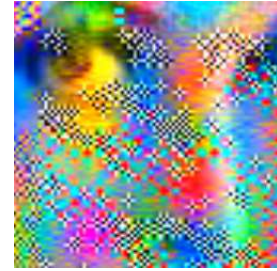

Received JPWL

image: $14.98 \mathrm{~dB}$

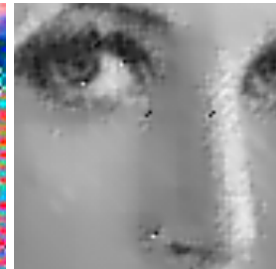

Received LAR image: $22.08 \mathrm{~dB}$

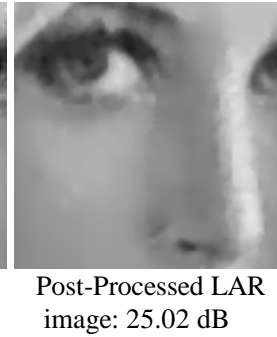

Figure 4: LAR and JPWL image transmission over the COST207 TU channel SNR $=19 \mathrm{~dB}, \mathrm{f}_{\mathrm{D}} \times \mathrm{T}_{\mathrm{S}}=0.001$, $\mathrm{BER}=1.6 \times 10^{-2}$.

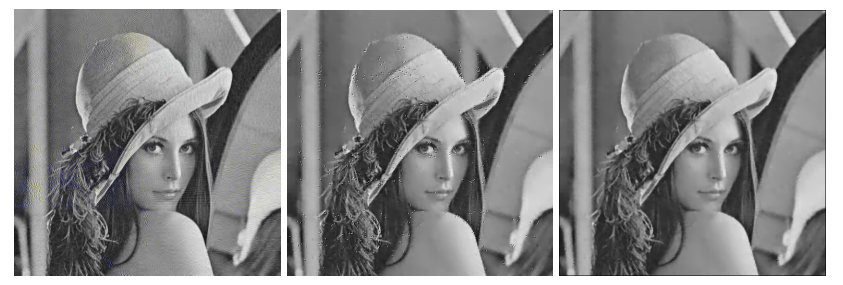

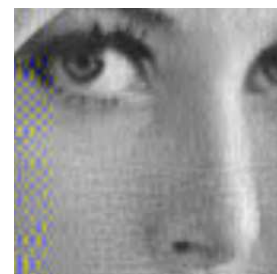

Received JPWL image: $27.47 \mathrm{~dB}$

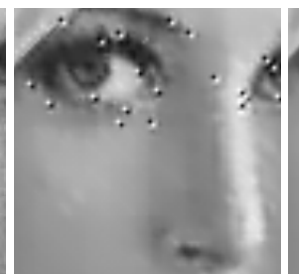

Received LAR image: $27.18 \mathrm{~dB}$

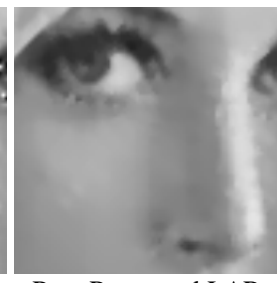

Post-Processed LAR image: $25.91 \mathrm{~dB}$
Figure 5: LAR and JPWL image transmission trough the COST207 TU channel SNR $=21.5 \mathrm{~dB}, \mathrm{f}_{\mathrm{D}} \times \mathrm{T}_{\mathrm{s}}=0.001, \mathrm{BER}=8 \times 10^{-3}$.

\section{REFERENCES}

[1] G. Sherwood and K. Zeger, "Error Protection for Progressive Image Transmission Over Memoryless and Fading Channels," IEEE Trans. Communications, vol. 46, no. 12, pp.1555-1559, December 1998.

[2] N. Thomos, N. V. Boulgouris, and M. G. Strintzis, "Wireless Image Transmission Using Turbo Codes and Optimal Unequal Error Protection”, IEEE Int. Conf. on Image Processing, Barcelona, Spain, Vol. 1, pp.73-76, Sep. 2003.

[3] N. Thomos, N. V. Boulgouris, and M. G. Strintzis, "Wireless transmission of images using JPEG2000", IEEE Int. Conf. on Image Processing, Singapore, pp. 2523-2526, Oct. 2004.

[4] "JPEG 2000 image coding system - Part 11: Wireless JPEG 2000 Final Draft International Standard", ISO/IEC JTC1/SC29/WG1WG1N3819,Nov.2005.

[5] F. Dufaux and D. Nicholson, "JPWL: JPEG 2000 for wireless applications", in SPIE Proc. Applications of Digital Image Processing XXVII, Denver, CO, Aug. 2004.

[6] F. Dufaux, G. Baruffa, F. Frescura, D. Nicholson, "JPWL - an extension of JPEG 2000 for wireless imaging”, in IEEE Proc. Int. Symp. on Circuits and Systems (ISCAS), Island of Kos, Greece, May 2006. 
[7] O. Déforges, M. Babel, L. Bédat, and J. Ronsin, "LAR codec: a representation and image compression scheme based on adaptive resolution scheme and self-extracting region representation," IEEE Trans. on Circuits and Systems for Video Technology 17(8), pp 974-987, August 2007.

[8] M. Paetzold, Mobile fading channels, Wiley, 2002.

[9] R. H. Clarke, "A statistical theory of mobile-radio reception", Bell Syst. Tech. Journal, vol 47, pp. 957-1000, July/Aug. 1968.

[10] COST 207, "Digital land mobile radio communications", Office for Official Publications of the European Communities, Final Report, Luxembourg, 1989.

[11] Svensson, N.A.B. On differentially encoded star 16QAM with differential detection and diversity Vehicular Technology, IEEE Transactions on Volume 44, Issue 3, Aug. 1995 Page(s):586 - 593

[12] M. Babel, O. Déforges and Joseph Ronsin, "Interleaved S+P Pyramidal Decomposition with Refined Prediction Model", Proc. of IEEE International Conference on Image Processing, ICIP'05, Genova, Italy, pp 750-753, Sept. 2005.

[13] J. Motsch, O. Déforges and Marie Babel, "Embedding Multilevel Image Encryption in the LAR Codec", Proc. of IEEE Communications International Conference 06, June 2006.

[14] C. Chatellier, H. Boeglen, C. Perrine, C. Olivier, O Haeberlé, A robust joint source channel coding scheme for image transmission over the ionospheric channel, ELSEVIER SPIC, August 2007. 